\title{
FAST PARAMETRIC SNAKES FOR 3D MICROSCOPY
}

\author{
Ricard Delgado-Gonzalo ${ }^{\star}$,Nicolas Chenouard ${ }^{\star \dagger}$, and Michael Unser ${ }^{\star}$ \\ ${ }^{\star}$ Biomedical Imaging Group, École polytechnique fédérale de Lausanne (EPFL), Switzerland \\ $\dagger$ Center for Biomedical Imaging (CIBM), Université de Lausanne (UNIL), Switzerland
}

\begin{abstract}
We present a new fast active contour for images in 3D microscopy. We introduce a fully parametric design that relies on exponential B-spline bases and allows us to impose a sphere-like topology. The proposed 3D snake can approximate blob-like objects with good accuracy. The optimization process is remarkably fast. Our technique yields successful segmentation results even for a challenging data set where object contours are not well defined. This happens because our parametric approach allows us to favor prior shapes. This work comes with a companion software that allows extensive interactions between the end-user and our snakes through the intuitive manipulation of the few control points that fully characterize them.
\end{abstract}

Index Terms-Active contours, segmentation, 3D, parameterization, microscopy.

\section{INTRODUCTION}

Three-dimensional (3D) active contours (a.k.a. snakes) are popular segmentation tools in 3D biomedical images. They provide accurate shape detection while enforcing object integrity. Implicit methods based on a level-set formulation of the Chan-and-Vese problem have been investigated extensively over the past decade [1]. Some effort was invested to obtain semi-parametric approaches using simplex meshes [2], and, more recently, 3D triangular meshes [3]. However, fully parametric snakes still need to be worked out in 3D. In this paper, we propose the first 3D spline-based parametric snakes for the analysis of images in 3D microscopy.

We propose a continuous model of the $3 \mathrm{D}$ snake surface that is parameterized by few control points by using exponential B-splines. The framework is versatile. By contrast with most implicit techniques, it allows one to impose prior-shape constraints to the snake. In particular, we propose a model for outlining ellipsoidal objects and sphere-like closed surfaces that are often met in cell biology. This is an extension to 3D of the work in [4] whereby the splinebased parameterization allows us to derive a fast algorithm. This is crucial for biological applications such as cell tracking in timelapse sequences of 3D images, which produce tremendous amounts of data. We have investigated the efficiency of the proposed approach with the analysis of several sets of real microscopic images and demonstrated real-time performance.

The parametric approach for the design of 3D snakes also allows us to design a novel type of semi-automatic 3D segmentation scheme in which user interaction plays an important role. Because the snake is fully parameterized by only few control points in space, the user is able to easily guide and modify the snake by interacting with anchors in dedicated 2D and 3D image views which also feature a live

This work was funded by the Swiss SystemsX.ch initiative under Grant 2008/005 and the Swiss National Science Foundation under Grant 200020121763.

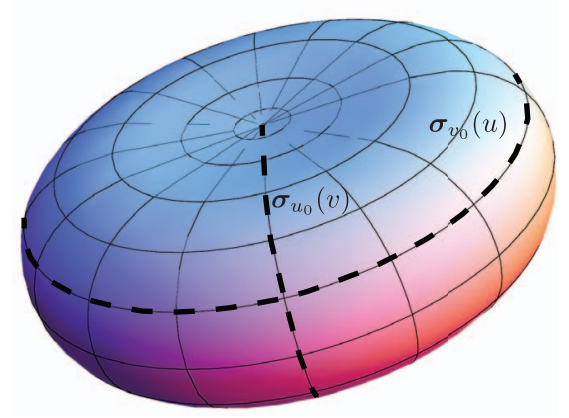

Fig. 1. Rendering of the snake surface.

display of the snake. This ability is precious for busy biological environments which may require user input and feedback. The software implementing our techniques is provided as an open-source library in an effort to provide useful tools to the bioimaging community.

\section{3D SNAKE MODEL}

We define our 3D model for a snake as a closed parametric surface $\sigma(u, v)$, where $u$ and $v$ are arbitrary continuous parameters. The bivariate coordinate functions $x(u, v), y(u, v)$, and $z(u, v)$ are efficiently parameterized by linear combinations of suitable basis functions and an arbitrary sequence of vector control points $\{\mathbf{c}[i, j]\}_{i, j \in \mathbb{Z}}$. Among all possible bases, we focus on those derived from the tensor product of a compactly supported generator

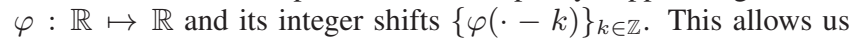
to take advantage of the availability of fast and stable interpolation algorithms [5]. Then, the parametric representation of the surface is given by the vectorial equation

$$
\boldsymbol{\sigma}(u, v)=\sum_{i=-\infty}^{\infty} \sum_{j=-\infty}^{\infty} \mathbf{c}[i, j] \varphi(M u-i) \varphi(M v-j),
$$

where $\varphi$ determines the shapes the parametric surface can adopt, and the integer $M$ fixes the degrees of freedom of the model.

Using an earth simile for our sphere-like snake, the curves that are obtained when fixing the second parameter in (1) correspond to the circles of latitude shown in Figure 1. Formally, the functions $\boldsymbol{\sigma}_{v_{0}}(u)=\boldsymbol{\sigma}\left(u, v_{0}\right)$ for all $v_{0}$ have to be periodic in $u$ in order to generate closed curves. In order to satisfy this condition, it is necessary to apply periodic boundary conditions along the first index of the sequence of control points. Therefore, the sequence of coefficients becomes $M$-periodic and satisfies $\mathbf{c}[i, j]=\mathbf{c}[i+M, j]$. For simplicity, in (1) we normalized this period to be unity. Under 
these conditions, we can reduce the first infinite summation in (1) to a finite one involving periodized basis functions as

$$
\boldsymbol{\sigma}_{v_{0}}(u)=\sum_{i=0}^{M-1} \sum_{j=-\infty}^{\infty} \mathbf{c}[i, j] \varphi_{M}(M u-i) \varphi\left(M v_{0}-j\right),
$$

where $\varphi_{M}(\cdot)=\sum_{n=-\infty}^{\infty} \varphi(\cdot-M n)$ is the $M$-periodization of $\varphi$. This reorganization of the terms is computationally efficient [6].

Using this model, $\sigma_{v_{0}}(u)$ can approximate any closed curve as accurately as desired by increasing the value of $M$ [5]. We choose $\varphi$ to be the basis function made of exponential B-splines we designed in [4], with

$$
\varphi(t)= \begin{cases}\frac{\cos \frac{2 \pi|t|}{M} \cos \frac{\pi}{M}-\cos \frac{2 \pi}{M}}{1-\cos \frac{2 \pi}{M}} & 0 \leq|t|<\frac{1}{2} \\ \frac{1-\cos \frac{2 \pi(3 / 2-|t|)}{M}}{2\left(1-\cos \frac{2 \pi}{M}\right)} & \frac{1}{2} \leq|t|<\frac{3}{2} \\ 0 & \frac{3}{2} \leq|t| .\end{cases}
$$

It has a quadratic approximation rate, allows the snake to perfectly replicate elliptic shapes, and the size of its support is the shortest possible to satisfy this two conditions [6]. This results in a maximally efficient scheme.

Moreover, following our earth simile, the curves that are obtained when fixing $u$ in (1) correspond to meridians. Formally, the curves $\boldsymbol{\sigma}_{u_{0}}(v)=\boldsymbol{\sigma}\left(u_{0}, v\right)$ originate at the north pole $\boldsymbol{c}_{\mathrm{N}}$ and end at the south pole $\boldsymbol{c}_{\mathrm{S}}$. Therefore, $\boldsymbol{\sigma}_{u_{0}}(0)=\boldsymbol{c}_{\mathrm{N}}$ and $\boldsymbol{\sigma}_{u_{0}}(1)=\boldsymbol{c}_{\mathrm{S}}$. By convention, the parameter range is normalized to lie within $[0,1]$.

It has been shown that, under the appropriate boundary conditions on $\boldsymbol{\sigma}_{u_{0}}(v)$, the parameterization of the poles of a pseudosphere is well defined [7]. In our case, it is necessary to impose conditions on the vector coefficients. This is solved by identifying all control points at the extreme of the parameterization with a multiplicity equal to the length of the support of $\varphi$, so that

$$
\begin{aligned}
\boldsymbol{c}_{\mathrm{N}} & =\boldsymbol{c}[-1, j]=\boldsymbol{c}[0, j]=\boldsymbol{c}[1, j] \\
\boldsymbol{c}_{\mathrm{S}} & =\boldsymbol{c}[M-1, j]=\boldsymbol{c}[M, j]=\boldsymbol{c}[M+1, j] \quad \forall j .
\end{aligned}
$$

Finally, we can rewrite the complete parametric form of our snake using (2) and (3) as

$$
\begin{aligned}
\boldsymbol{\sigma}(u, v)= & \sum_{i=0}^{M-1} \sum_{j=2}^{M-2} \mathbf{c}[i, j] \varphi_{M}(M u-i) \varphi(M v-j) \\
& +\boldsymbol{c}_{\mathrm{N}} \sum_{j=-1}^{1} \varphi(M v-j) \\
& +c_{\mathrm{S}} \sum_{j=-1}^{1} \varphi(M(v-1)-j),
\end{aligned}
$$

which leaves a total of $M(M-3)+2$ free control points.

As the parameterization of our snake is explicit, it is straightforward to perform a pointwise analysis on its surface. To do so, we define tangent planes by their tangent vectors $\mathbf{T}_{u}=\frac{\partial \sigma}{\partial u}$ and $\mathbf{T}_{v}=\frac{\partial \sigma}{\partial v}$. Then, the normal vectors to the snake are $\mathbf{n}=\mathbf{T}_{u} \wedge \mathbf{T}_{v}$. Note that these normal vectors are all pointing either inwards or outwards, depending on the initialization.

\section{FAST ACTIVE CONTOUR OPTIMIZATION}

\subsection{Image Energy}

Active contours are driven by an energy function that determines the quality of the segmentation. Often, traditional snakes rely on edge maps to guide them. The most popular approach is based on the magnitude $\|\nabla f\|$ of the gradient [8]. Mathematically, they minimize

$$
E_{\mathrm{mag}}=-\oiint_{S}\|\nabla f\| \mathrm{d} S
$$

where the integration is performed on the closed surface $S$ of the contour. The major drawback of this approach is that the snake get distracted by nearby targets.

We solve this issue by remembering that our goal is to segment a closed volume. The parameterization (4) offers us the opportunity to choose the orientation of the normal vectors at initialization time. From now on, and without loss of generality, we assume it to be outwards. When the energy reaches its minimum, we expect the directions of the image gradients to be the same as the directions of the normal vectors. Assuming that we want to segment a dark object surrounded by a brighter region, we finally propose the following image energy:

$$
E_{\text {grad }}=-\oiint_{S} \nabla f \cdot \mathbf{d} \mathbf{S}=-\oiint_{S}\left(\nabla f \cdot \frac{\mathbf{n}}{\|\mathbf{n}\|}\right) \mathrm{d} S .
$$

A similar scheme was proposed in 2D in [9], where it was shown that energies defined by an integral over the boundary of a snake can be expressed as an integrals over the region enclosed by the snake. This allows for the easy combination of energies based on edge maps with region energies. In particular, (5) can be rewritten using the Gauss (or divergence) theorem as

$$
E_{\text {grad }}=-\iiint_{V} \operatorname{div}(\nabla f) \mathrm{d} V=\iiint_{V} \underbrace{-\Delta f}_{f_{\Delta}} \mathrm{d} V,
$$

where $V$ is the volume enclosed by $S$, and $f_{\Delta}$ can be precomputed.

\subsection{Efficient Implementation}

The computational cost of the evolution of the snake is dominated by that of the volume integral in (6). An efficient way to implement these operations is the use of pre-integrated images. By the Gauss theorem, we rewrite the volume integral as the surface integral

$$
\iiint_{V} f_{\Delta} \mathrm{d} V=\oiint_{S} f_{\Delta}^{y} \mathrm{~d} z \wedge \mathrm{d} x
$$

where $f_{\Delta}^{y}(x, y, z)=\int_{-\infty}^{y} f_{\Delta}(x, \tau, z) \mathrm{d} \tau$. The use of the Gauss theorem to rewrite volume integrals as surface integrals reduces the computational load dramatically. This can only be achieved if the surface is defined continuously, like in (1).

Since the number $M(M-3)+2$ of parameters that define the snake is relatively small, the optimization can be efficiently carried out by Powell-like line-search methods which require the derivatives of the energy function with respect to the parameters (i.e., the knot coefficients), and converge quadratically to the solution.

\subsection{Semi-Automatic Segmentation with User Interactions}

Our 3D segmentation technique has been programmed as an opensource plugin for the multi-platform software ICY [10] and is available on the official plugin repository.

By contrast with many other implicit and global parametric snakes, only a few control points are sufficient to fully parameterize our proposed snake, which eases the interactions with the user. This has encouraged us to develop a dedicated graphical user interface 

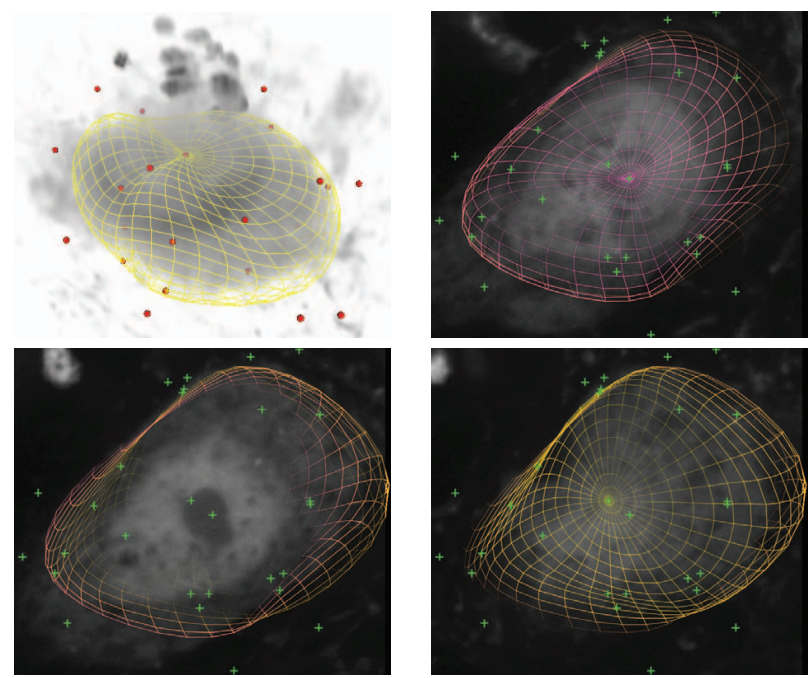

Fig. 2. Segmentation of a cell in a 3D confocal image of a murine brain (image courtesy of Sabine Scheibe and Sebastian Rhode at TILL Photonics). Top-left: 3D view of the segmented glomeruli. From left to right and top to down: snake at different $z$-axis positions of the $3 \mathrm{D}$ volume overlaid to the original 2D images.

that lets the user initialize the 3D snake position, and trim it even after the optimization process. As illustrated in Figures 2 and 3, the user can intuitively manipulate the position of any point by selecting it in either the 2D or 3D mesh representations of the snake. The $2 \mathrm{D}$ view also provides $3 \mathrm{D}$ cues as we color the mesh depending on the $z$-axis coordinate and set its transparency to match the distance to the slide of the volume which is displayed. Editing the points is performed with simple mouse actions. The live update of the $2 \mathrm{D}$ and $3 \mathrm{D}$ views of snake is very fast. This is due to the fact that the change in one parameter affects the structure locally. Thus, just a limited region of the snake surface has to be recomputed. As a result, a fast and intuitive semi-automatic segmentation procedure that loops between snake initialization, optimization, and correction, is made possible.

\section{EXPERIMENTS}

We present two experimental setups. In the first one, we work with synthetic data and perform an objective validation. In the second one, we illustrate some real applications of our snake.

\subsection{Accuracy}

We generated 7 reference volumes $(256 \times 256 \times 256)$ by voxelwise sampling of the combination of two spheres of radius 50 pixel. We illustrate these shapes in Table 1. They are parameterized with the distance $d$, in pixel units, between the centers of the spheres. For $d<0$, the shape is built by the intersection of the two spheres. For $d \geq 0$, the shape is built by the convex hull of their union. The grayscale values of the images are 255 for the shape and 0 for the background. We used the Jaccard similarity index $J$ to measure the performance of the snake.

\begin{tabular}{c|ccccc}
\hline & $d$ & $M=4$ & $M=5$ & $M=6$ & $M=7$ \\
\hline & -30 & 0.92 & 0.92 & 0.91 & 0.91 \\
-20 & 0.94 & 0.95 & 0.94 & 0.95 \\
-10 & 0.95 & 0.97 & 0.96 & 0.97 \\
0 & 0.94 & 0.98 & 0.96 & 0.97 \\
10 & 0.90 & 0.97 & 0.96 & 0.96 \\
& & 0.88 & 0.89 & 0.93 & 0.92 \\
& 30 & 0.85 & 0.93 & 0.93 & 0.93 \\
\hline
\end{tabular}

Table 1. Jaccard similarity index of our snake for synthetic data.

In Table 1, we investigated the dependence of $J$ on $M$ and on the distance $d$ between the spheres. Our results show that the segmentation performance is consistently very high: $J$ ranges from 0.85 to 0.98 , which demonstrates the ability of the proposed model to segment objects with an ellipsoidal-like topology. For compact objects $(d \leq 0)$, the models of low order $(M=4)$ provide a good approximation $(J \geq 0.92)$ of the target object and a model of higher order is not required. For elongated objects $(d>0)$, the use of a model of high order is beneficial to the segmentation performance as the increased degrees of freedom allow for an improvement up to 0.08 when $M$ increases from 4 to 7 .

\subsection{Segmentation of 3D Confocal Microscopic Images}

\subsubsection{Cell-Body Segmentation}

We processed a stack $(576 \times 504 \times 200)$ of confocal $(\times 60)$ images from the brain cortex of a rat, with YFP labeling for the neurons and GFP for the microglia ${ }^{1}$. The challenge was to accurately segment the bodies of the neuronal cell despite their non-spherical shape, the lack of clear borders, and the presence of several surrounding objects. We set $M=7$, roughly initialized the snake position around each cell body as a sphere, and run the optimization process until convergence. One example of resulting segmentation is shown in Figure 2. It shows that the snake was able to adapt remarkably well to the $3 \mathrm{D}$ cell shape: the surface is accurately fitted despite the limited degrees of freedom of the model, while irregularities are properly smoothed out. The optimization process took only $0.74 \mathrm{~s}$, which is faster than the duration of the acquisition of such data (usually much less than $1 \mathrm{~Hz})$.

\subsubsection{Segmentation of Glomeruli}

We investigated the segmentation of olfactory glomeruli in the mouse brain, that represent neuroglial functional units in olfactory information processing [11]. With a Topro staining, glomeruli correspond to the dark areas delimited by fluorescent cell bodies. In Figure 3, they are visualized as bright areas with the inverted look-up-table. However, the surrounding fluorescent cells do not form continuous boundaries which penalizes nonparametric activecontour as the snake may 'leak out' between the neighboring cells. By contrast, we show in Figure 3 that the proposed snake method (with $M=7$ ) is able to accurately identify the glomerulus border.

\footnotetext{
${ }^{1}$ http://www.cellimagelibrary.org/images/27155/
} 

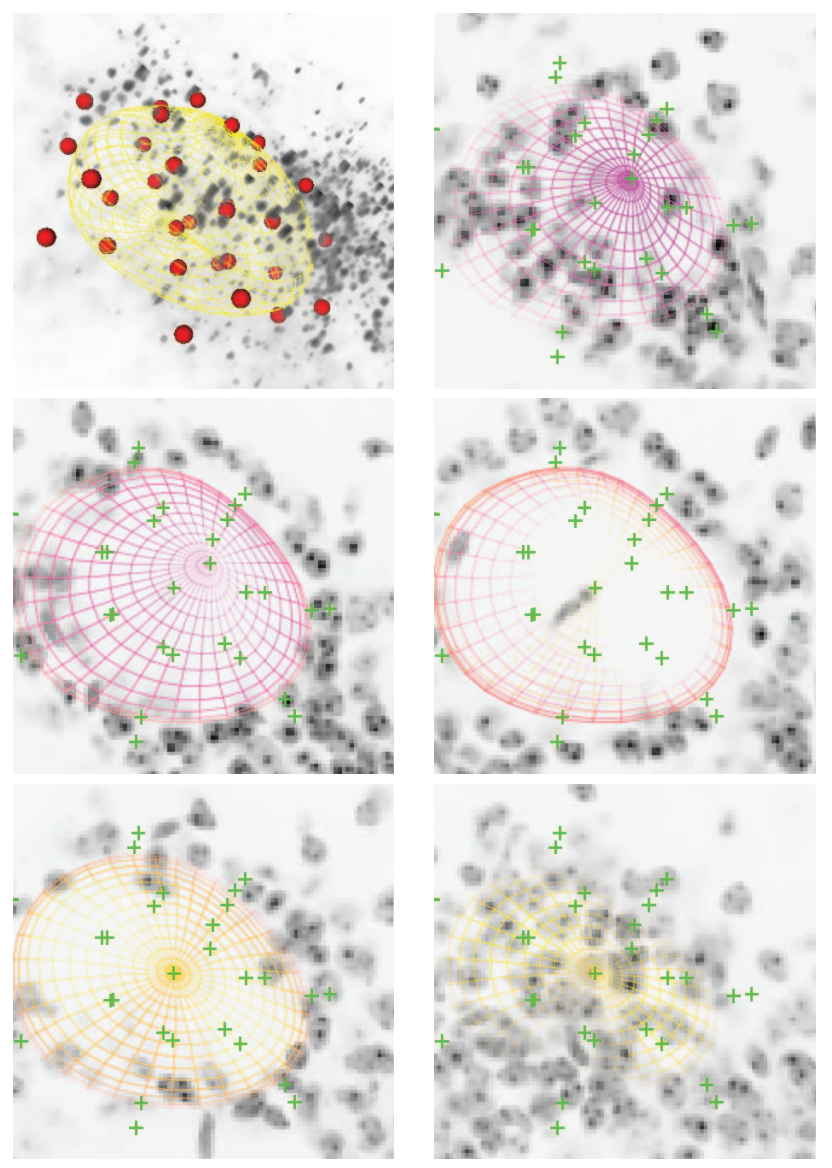

Fig. 3. Segmentation of an olfactory glomerulus in a 3D confocal image $(256 \times 256 \times 67)$ of a mouse brain (inverted colors, image courtesy of Lisa Roux at the Collège de France). Top-left: 3D view of the segmented glomeruli. From left to right and top to down: snake at different $z$-axis positions $(z=3,10,17,24$ and $31 \mu \mathrm{m})$ of the $3 \mathrm{D}$ volume overlaid to the original $2 \mathrm{D}$ images.

This is an key advantage of the proposed method that allows one to exert one an a priori control over the snake regularity and topology through the design of the parametric model. Here again, the optimization process was remarkably fast and lasted $1.74 \mathrm{~s}$.

\section{CONCLUSION}

Our contribution in this paper is a new fully parametric snake with a sphere-like topology built upon exponential B-splines, and an edgebased energy functional capable of maintaing the consistency of the segmentation in the presence of clutter. Our snake is characterized by fewer control points than nonparametric snakes and can approximate any sphere-like surface with arbitrary precision. The modification of one control point affects a limited region of the snake surface, which results in intuitive interactions with the user. This is made possible by a parameterization based on splines. We were able to accelerate the implementation by taking advantage of Gauss' theorem, which was facilitated by the availability of explicit expressions of our bases.

We have applied our snakes to a variety of problems that involve synthetic simulations, and challenging real datasets where the object contours were not well defined. As a result, various experiments have shown that the proposed 3D snake can approximate with good accuracy blob-like objects. Moreover, the optimization process is remarkably fast. It may consequently open the way to real-time applications.

\section{REFERENCES}

[1] T. Chan and L. Vese, "Active contours without edges," IEEE Transactions on Image Processing, vol. 10, pp. 266-277, February 2001.

[2] H. Delingette, "General object reconstruction based on simplex meshes," International Journal of Computer Vision, vol. 32, pp. 111-146, September 1999.

[3] A. Dufour, R. Thibeaux, E. Labruyere, N. Guillen, and J.-C. Olivo-Marin, "3-D Active meshes: Fast discrete deformable models for cell tracking in 3-D time-lapse microscopy," IEEE Transactions on Image Processing, vol. 20, pp. 1925-1937, July 2011.

[4] R. Delgado-Gonzalo, P. Thévenaz, C. Seelamantula, and M. Unser, "Snakes with ellipse-reproducing property," IEEE Transactions on Image Processing, 2012. doi:10.1109/TIP.2011.2169975.

[5] M. Jacob, T. Blu, and M. Unser, "Sampling of periodic signals: A quantitative error analysis," IEEE Transactions on Signal Processing, vol. 50, pp. 1153-1159, May 2002.

[6] R. Delgado-Gonzalo, P. Thévenaz, and M. Unser, "Exponential splines and minimal-support bases for curve representation," Computer Aided Geometric Design, vol. 29, pp. 109128, February 2012.

[7] L. Schumaker and C. Traas, "Fitting scattered data on spherelike surfaces using spherical splines," Numerische Mathematik, vol. 60, pp. 133-144, January 1991.

[8] M. Kass, A. Witkin, and D. Terzopoulos, "Snakes: Active contour models," International Journal of Computer Vision, vol. 1, pp. 321-331, January 1988.

[9] M. Jacob, T. Blu, and M. Unser, "Efficient energies and algorithms for parametric snakes," IEEE Transactions on Image Processing, vol. 13, pp. 1231-1244, September 2004.

[10] F. de Chaumont, S. Dallongeville, and J.-C. Olivo-Marin, "ICY: A new open-source community image processing software," in Proceedings of the 8th IEEE International Symposium on Biomedical Imaging: From Nano to Macro (ISBI'11), (Chicago, USA), pp. 234-237, March 30-April 2, 2011.

[11] L. Roux, K. Benchenane, J. Rothstein, G. Bonvento, and C. Giaume, "Plasticity of astroglial networks in olfactory glomeruli," Proceedings of the National Academy of Sciences of the United States of America, vol. 108, no. 45, pp. 1844218446, 2011. 